\title{
Mitigating the scourge of non-alcoholic fatty liver disease in Ghana
}

\author{
Ghana Med J 2017; 51(3): 98-100 DOI: http://dx.doi.org/10.4314/gmj.v51i3.1
}

\section{INTRODUCTION}

Non-alcoholic fatty liver disease (NAFLD) is fast becoming the leading cause of liver disease in Western countries, affecting $25 \%$ of the general population ${ }^{1}$ and increasing to $90 \%$ of obese individuals with diabetes and other features of the metabolic syndrome. ${ }^{2}$ Moreover, its prevalence is expected to continue to rise in parallel with the obesity epidemic due to the adoption of sedentary lifestyle and increased consumption of high fat diet. ${ }^{3}$ In the United States, NAFLD is expected to become the leading indication for liver transplantation (LT) within the next 10 years. ${ }^{4}$ NAFLD is currently considered to be a major health burden in developed countries and is gradually increasing in developing countries due to urbanization and change in lifestyle habits, particularly in the countries of Arabian Gulf (with the highest incidence in the world), but it cannot be ignored in West Africa, where the problem is becoming steadily more prevalent.

Primary liver cancer is a major complication of liver disease and a contributor to global cancer incidence and mortality. ${ }^{5}$ Worldwide, liver cancer is the fifth most common cancer and the second most frequent cause of cancer death in men, while in women it is the seventh most commonly diagnosed cancer and the sixth leading cause of cancer death. ${ }^{6}$ Major risk factors for hepatocellular carcinoma (HCC) include hepatitis B virus (HBV) and hepatitis $\mathrm{C}$ virus (HCV) chronic infections, alcoholic liver disease, and NAFLD. ${ }^{7}$ Several population-based studies conducted in various geographic areas have recorded a significantly increased incidence of HCC in patients with diabetes and obesity. ${ }^{8-11}$ There is also growing evidence that suggests an increased risk of HCC in NAFLD patients. ${ }^{12-14}$ Under these circumstances, NAFLD-related HCC incidence is expected to increase in the future. Ghana is a developing country, with limited access to regular screening and palliative care, prevalent comorbidities (e.g. Hepatitis B, HIV/AIDS), and risk factors (e.g. Alcohol use) all playing a role, the scourge of fatty liver disease cannot be overlooked. NAFLD is currently considered a major health burden in developed countries and is gradually increased in developing countries due to urbanization and change in lifestyle habits.

\section{Fatty liver}

It is an umbrella of disorders defined by abnormal accumulation of triglycerides in the liver; and ranges from fatty liver alone (steatosis) to fatty liver with inflammation (steatohepatitis) ${ }^{15}$
It can occur with use of alcohol (alcohol-related fatty liver) or in the absence of alcohol (non-alcoholic fatty liver disease (NAFLD).

NAFLD is defined as the presence of $\geq 5 \%$ of hepatic steatosis (HS), in the absence of competing liver disease etiologies, such as chronic viral hepatitis, use of medications that induce steatosis and other chronic liver diseases, such as autoimmune hepatitis, hemochromatosis, Wilson's disease, or significant alcohol consumption. It is one of the commonest causes of abnormal liver function tests (LFTs). NAFLD is strongly associated with epidemic of obesity and type 2 diabetes mellitus (DM)16 and increasingly being recognised as the liver component of metabolic syndrome. ${ }^{17}$ Nonalcoholic steatohepatitis, a potentially serious form of the disease, is marked by liver inflammation, which may progress to scarring and irreversible damage. This damage is similar to the damage caused by heavy alcohol use. At its most severe, nonalcoholic steatohepatitis can progress to cirrhosis, liver failure and liver cancer. Obesity, type 2 diabetes mellitus (T2DM), and dyslipidemia are the most common metabolic risk factors associated with NAFLD. Among the components of metabolic syndrome, current evidence strongly indicates obesity and diabetes as hepatocellular carcinoma (HCC) risk factors. There is also growing evidence that suggests an increased risk of HCC in NAFLD patients, even surpassing other aetiologies in some high-income countries. Epidemiologic data demonstrate a parallel rise in prevalence of obesity, diabetes, NAFLD, and HCC. As obesity and its related diseases have steadily afflicted larger populations, HCC incidence is expected to increase in the future. Pathophysiologic mechanisms that underlie NAFLD development and subsequent progression to nonalcoholic steatohepatitis and cirrhosis (insulin resistance and hyperinsulinemia, oxidative stress, hepatic stellate cell activation, cytokine/adipocytokine signalling pathways, and genetic and environmental factors) appear to play a significant role in the development of NAFLD-related HCC. However, a comprehensive view of molecular mechanisms linking obesity, T2DM, and NAFLD-related HCC, as well as the exact sequence of molecular events, is still not understood in its entirety.

\section{Mitigating the scourge of NAFLD}

Assessing and management of NAFLD can be a daunting task; NAFLD is prevalent in people in their 5th decade of life who often have multiple comorbidities such as obesity, hypertension, hyperlipidaemia and diabetes. 
Improving provision of assessment and testing of those individuals at highest risk of developing liver disease, creating awareness and understanding of liver disease among primary and community care, and local government partners to help detect early liver disease and make appropriate referral will go a long way in preventing late presentation of the disease with substantial morbidity and high mortality rates.

A key challenge in the clinical care of NALFD patients in Ghana is the lack of safe, reliable, and cost-effective means of diagnosing and staging the disease. Fatty liver is frequently discovered incidentally during ultrasound, CT or MRI scanning and a proportion of these individuals may have advanced liver disease in spite of having normal LFTs. Currently, referral practices of these individuals are patchy and development of a robust risk assessment and referral guideline should be implemented. Liver fibrosis is believed to be the most important factor for progression to cirrhosis ${ }^{18}$, but as yet there is no ideal tool for assessing its presence. Ultrasound imaging may pick up fatty liver disease if about $30 \%$ of the liver is filled with fat making it unreliable. Liver biopsy remains the gold standard for both diagnosis and staging of the disease, but is not without risk. Although reliable, the process is costly requires significant expertise and it is associated with complications such as bleeding and not insignificant pain even in the best hands. Non-invasive, safe, low-cost, and highly reliable imaging techniques have been developed such as ultrasound-based transient elastography using the FibroScan machine (which is readily accepted by patients and providers). These hold tremendous promise in the clinical care of these patients around the world,but fibroscan is currently non-existent in Ghana..

Additionally, treatment decisions made based on liver function tests fail to capture disease severity in NAFLD. ${ }^{19}$ There are currently no approved pharmacological interventions for NAFLD, and current management strategies aim to identify and treat features of the metabolic syndrome, in order to reduce long-term cardiovascular and liver-related morbidity and mortality. ${ }^{20}$ Features of the metabolic syndrome should be aggressively managed, with a focus on weight loss. This is difficult to achieve in this population; even in highly supervised randomized clinical trials (RCTs), less than $50 \%$ participants have been shown to achieve the ideal $7 \%$ total body weight reduction. ${ }^{21}$ Tackling rising obesity levels requires a multi-faceted approach involving a range of activities including behavior change and access to weight management services. Changes to lifestyle, such as eating a healthy, balanced diet and regular physical activity, can help reduce levels of obesity (particularly with reduction in carbohydrate content such as bread, rice, fufu and potatoes). Exercise as part of a healthy lifestyle has proven benefits to patients suffering from liver disease. There are several opportunities for public health nutrition and dietetic interventions to be put in place to support the prevention and reduction of obesity. The development of a locally agreed care pathway for patients with abnormal liver function tests and risk assessment of those incidentally found to have fatty liver disease. A simple and concise guidance to interpret abnormal liver function tests tool should be developed by the hepatologists for use in primary care, and steps taking to ensure its widespread use in all practices within the country. Opportunistic advice on lifestyle changes including dietary advice and weight reduction in the appropriate individual should also be provided in all settings. Obesity services and diabetology clinics should be involved to support the early detection and treatment of liver inflammation associated with fatty liver disease by referring appropriate patients.

\section{CONCLUSION}

NAFLD is a very common condition affecting approximately $30 \%$ of the population and can cause significant liver disease in a significant proportion of patients. Accurate diagnosis and staging is important in determining the appropriate long-term management for patients with NAFLD. To mitigate this scourge, a dedicated onestop-shop multidisciplinary fatty liver clinic is a necessity in our environment. This should tackle the NAFLD problem from a 360 degree viewpoint and should involve different specialist services, including hepatologist, diabetologists, radiologists, dietician, psychologist, physical therapist, and nurses trained in liver care. Such a clinic can provide expert management targeted towards those at highest risk of progressive liver disease, as well as access to emerging diagnostics and clinical trials.

\section{Dr. Adwoa Agyei-Nkansah MB ChB, MACP, FWACP \\ Lecturer, Department of Medicine, University of Gha- na School of Medicine and Dentistry, P.O Box 4236, Accra. \\ E-mail: afrakomaagyei@yahoo.com \\ Conflict of interest: None declared}

\section{REFERENCES}

1. Armstrong MJ, Houlihan DD, Bentham L, et al: Presence and severity of non-alcoholic fatty liver disease in a large prospective primary care cohort. $J$ Hepatol 2012; 56: 234-240.

2. 2. Smits MM, Ioannou GN, Boyko EJ, et al: Nonalcoholic fatty liver disease as an independent manifestation of the metabolic syndrome: results of a US national survey in three ethnic groups. $J$ Gastroenterol Hepatol 2013; 28: 664-670. 
3. Visscher TL, Heitmann BL, Rissanen A, et al: A break in the obesity epidemic? Explained by biases or misinterpretation of the data? Int J Obes (Lond) 2015; 39: 189-198.

4. Wong RJ, Aguilar M, Cheung R, et al: Nonalcoholic steatohepatitis is the second leading etiology of liver disease among adults awaiting liver transplantation in the United States. Gastroenterology 2015; 148: 547-555.

5. McGlynn KA, London WT. The global epidemiology of hepatocellular carcinoma: present and future. Clin Liver Dis. 2011;15:223-43, vii-x.

6. Jemal A, Bray F, Center MM, Ferlay J, Ward E, Forman D. Global cancer statistics. CA Cancer $J$ Clin. 2011;61:69-90.

7. El-Serag HB. Hepatocellular carcinoma. $N$ Engl $J$ Med. 2011;365:1118-1127.

8. Haslam DW, James WP. Obesity. Lancet. 2005;366:1197-1209.

9. Renehan AG, Tyson M, Egger M, Heller RF, Zwahlen M. Body-mass index and incidence of cancer: a systematic review and meta-analysis of prospective observational studies. Lancet.2008; 371:569-578.

10. Nair S, Mason A, Eason J, Loss G, Perrillo RP. Is obesity an independent risk factor for hepatocellular carcinoma in cirrhosis? Hepatology. 2002;36:150-155.

11. Koh WP, Wang R, Jin A, Yu MC, Yuan JM. Diabetes mellitus and risk of hepatocellular carcinoma: findings from the Singapore Chinese Health Study. Br J Cancer. 2013;108:1182-1188.

12. Koh WP, Wang R, Jin A, Yu MC, Yuan JM. Diabetes mellitus and risk of hepatocellular carcinoma: findings from the Singapore Chinese Health Study. Br J Cancer. 2013;108:1182-1188.

13. Marchesini G, Forlani G, Bugianesi E. Is liver disease a threat to patients with metabolic disorders? Ann Med. 2005;37:333-346.
14. White DL, Kanwal F, El-Serag HB. Association between nonalcoholic fatty liver disease and risk for hepatocellular cancer, based on systematic review. Clin Gastroenterol Hepatol. 2012;10:13421359.

15. Guerrero R, Vega GL, Grundy SM, Browning JD. Ethnic differences in hepatic steatosis: an insulin resistance paradox? Hepatology 2009; 49: 791-801.

16. Sears D. Fatty liver: Available at WWW.Medscape.com/ view article/588890 accessed 8/7/2010.

17. Chalasani N, Younossi Z, Lavine J, Diehl AM, Brunt E, Cusi K, et al. The diagnosis and management of non-alcoholic fatty liver disease: practice Guideline by the American Association for the Study of Liver Diseases, American College of Gastroenterology, and the American Gastroenterological Association. Hepatology 2012;55:2005-2023.

18. Angulo P, Kleiner DE, Dam-Larsen S, et al: Liver fibrosis, but no other histologic features, is associated with long-term outcomes of patients with nonalcoholic fatty liver disease. Gastroenterology 2015; 149: 389-397.

19. 19. Mofrad P, Contos MJ, Haque M, Sargeant C, Fisher RA, Luketic VA, et al: Clinical and histologic spectrum of nonalcoholic fatty liver disease associated with normal ALT values. Hepatology 2003; 37: 1286-1292.

20. Than NN, Newsome PN: Non-alcoholic fatty liver disease: when to intervene and with what. Clin Med (Lond) 2015; 15: 186-90.

21. Musso G, Cassader M, Rosina F, et al: Impact of current treatments on liver disease, glucose metabolism and cardiovascular risk in non-alcoholic fatty liver disease (NAFLD): a systematic review and meta-analysis of randomised trials. Diabetologia. 2012 Apr;55(4):885-904. doi: 10.1007/s00125-0112446-40 